\title{
SN-38-loaded nanofiber matrices for local control of pediatric solid tumors after subtotal resection surgery
}

Carles Monterrubio ${ }^{1,2}$, Guillem Pascual-Pasto ${ }^{1,2}$, Francisco Cano ${ }^{3}$, Monica VilaUbach $^{1,2}$, Alejandro Manzanares ${ }^{1,4}$, Paula Schaiquevich ${ }^{5}$, Jose A Tornero,6, Alejandro Sosnik ${ }^{7,8}$, Jaume Mora ${ }^{1,2}$, Angel M Carcaboso ${ }^{1,2^{*}}$

${ }^{1}$ Preclinical Therapeutics and Drug Delivery Research Program, Developmental Tumor Biology Laboratory, Fundació Sant Joan de Déu, Santa Rosa 39-57, Esplugues de Llobregat, 08950 Barcelona, Spain.

${ }^{2}$ Department of Pediatric Hematology and Oncology, Hospital Sant Joan de Déu Barcelona, Passeig Sant Joan de Déu 2, Esplugues de Llobregat, 08950 Barcelona, Spain

${ }^{3}$ Institut de Investigació Tèxtil i Cooperació Industrial de Terrassa (INTEXTER), Universitat Politecnica de Catalunya, Colom 15, Terrassa, 08222 Barcelona, Spain

${ }^{4}$ Department of Pediatric Surgery, Hospital Sant Joan de Déu Barcelona, Passeig Sant Joan de Déu 2, Esplugues de Llobregat, 08950 Barcelona, Spain ${ }^{5}$ CONICET-Clinical Pharmacokinetics Unit, Hospital de Pediatría JP Garrahan, Combate de los Pozos 1881, 1245 Buenos Aires, Argentina ${ }^{6}$ Cebiotex Biomedical Nanofibers, Parc Cientific de Barcelona, Baldiri i Reixac 4, 08028 Barcelona, Spain 
${ }^{7}$ Department of Materials Science and Engineering, Technion-Israel Institute of Technology, Technion City, Haifa, Israel

${ }^{8}$ Russell Berrie Nanotechnology Institute (RBNI), Technion-Israel Institute of Technology, Technion City, Haifa, Israel

"Corresponding author. Address: Fundació Sant Joan de Déu, Santa Rosa 3957, Esplugues de Llobregat, 08950 Barcelona, Spain. +34 936009751; amontero@fsjd.org (A.M. Carcaboso).

\begin{abstract}
In addition to surgery, local tumor control in pediatric oncology requires new treatments as an alternative to radiotherapy. SN-38 is an anticancer drug with proved activity against several pediatric solid tumors including neuroblastoma, rhabdomyosarcoma and Ewing sarcoma. Taking advantage of the extremely low aqueous solubility of $\mathrm{SN}-38$, we have developed a novel drug delivery system (DDS) consisting of matrices made of poly(lactic acid) electrospun polymer nanofibers loaded with SN-38 microcrystals for local release in difficultto-treat pediatric solid tumors. To model the clinical scenario, we conducted extensive preclinical experiments to characterize the biodistribution of the released SN-38 using microdialysis sampling in vivo. We observed that the drug achieves high concentrations in the virtual space of the surgical bed and
\end{abstract}


penetrates a maximum distance of $2 \mathrm{~mm}$ within the tumor bulk. Subsequently, we developed a model of subtotal tumor resection in clinically relevant pediatric patient-derived xenografts and used such models to provide evidence of the activity of the SN-38 DDS to inhibit tumor regrowth. We propose that this novel DDS could represent a potential future strategy to avoid harmful radiation therapy as a primary tumor control together with surgery.

\section{Keywords}

Local chemotherapy delivery, SN-38, poly(lactic acid) electrospun nanofibers, pediatric solid tumor, pharmacokinetics, microdialysis 


\section{Introduction}

Treatment of most malignant solid tumors in children relies on a combination of local control (surgery and radiation therapy; RT) and systemic chemotherapy [1]. Local tumor recurrence after resection surgery and RT remains a challenge. Despite local control of high-risk neuroblastoma, local tumor recurrences are developed in $10 \%$ of newly diagnosed patients and $50 \%$ of patients with locally persistent re-resected disease [2, 3]. Incidence of local recurrence after first complete remission in other pediatric malignancies such as Ewing sarcoma and primary localized rhabdomyosarcoma is $25 \%$ and $22 \%$, respectively $[4,5]$. The relevance of an adequate local control is underscored by the worse outcome observed in patients that develop local failure after initial complete remission [6]. The intensification of RT to improve local control after resection surgery is limited by unacceptable toxicity, especially in young children [7], and the increased risk of second malignancies [8]. In this context, new technology platforms are urgently called for to overcome the drawbacks associated with RT after tumor resection in children [9].

Polymeric drug delivery systems (DDSs) for the localized delivery of anticancer drugs emerged as one of the most promising approaches to treat resectable solid tumors [10]. Advantages of localized delivery comprise reduced systemic exposure to highly toxic agents and achievement of high local concentration of potent anticancer agents that are not suitable for systemic administration due to poor aqueous solubility $[10,11]$. However, the lack of comprehensive preclinical studies aiming to understand the pharmacokinetics of localized DDS in cancer still represents a significant hurdle towards a robust bench-to-bedside 
translation. One of the fundamental questions that remain unanswered is whether a substance locally released in the proximity of a solid tumor penetrates into the bulk of the malignant tissue or, conversely, the penetration is restricted to the tumor margins in direct contact with the DDS. To elucidate this, complex imaging techniques [12], radiation [13], or computer simulation [14] are usually required. In a previous study, we demonstrated the potential use of microdialysis to gain insight into these complex mechanisms in vivo [15].

SN-38 (10-hydroxy-campthothecin) in its lactone (active) form is a poorly soluble molecule that has shown potent preclinical activity against several pediatric solid tumors $[16,17]$. Irinotecan, the marketed soluble prodrug of SN38 , undergoes extensive conversion (>70\%) to SN-38 in nude mice [18], though it has demonstrated low clinical efficacy, likely due to only partial conversion (less than 10\%) into the active derivative upon systemic administration in patients [19]. In addition, SN-38 is rapidly hydrolyzed to an inactive carboxylate form in plasma. The encapsulation of $\mathrm{SN}-38$ into polymeric nanocarriers protected it from biodegradation and prolonged the half-life of the active form [20]. In this framework, SN-38 emerges as an optimal model anticancer drug to investigate the development of a novel DDS for application in the localized chemotherapy of pediatric solid tumors.

Electrospun polymer nanofiber matrices appear as one of the most versatile, reproducible and scalable nano-DDS [21]. They allow to adjust their size and shape to fill the space left by tumor resection, and provide a large surface area and porosity that facilitate the efficient release of the active cargo from the DDS to the tumor tissue [21]. Moreover, their monolithic nature eases manipulation, 
implantation and retention in the action body site, and prevents the characteristic migration of nanoparticles and microparticles.

Following this rationale, the present work reports for the first time on the development of a novel nanofiber DDS loaded with SN-38 microcrystals for the localized chemotherapy of pediatric solid tumors and the comprehensive characterization of the release rate, the in vivo localized biodistribution, the systemic pharmacokinetics and the antitumor activity in pediatric solid tumor models. A unique feature of the study is the use of microdialysis probes inserted in the tissue targeted by the localized release of SN-38 to quantify local drug levels at different depths in the tumor bulk or in the virtual space of the resection bed [15]. To our knowledge, such sampling technique has not yet been employed to monitor localized drug delivery in tumors.

\section{Materials and methods}

Reagents. SN-38 was obtained from Seqchem (Pangbourne, UK). Poly(lactic acid) (PLA) was from Velox, GmbH (Hamburg, Germany). Pluronic® F68 block polymer was a gift from BASF (Ludwigshafen, Germany). Irinotecan was purchased from Hospira (Lake City, IL, USA). 2-Hydroxypropyl-betacyclodextrin (HPBCD; molecular weight of $1400 \mathrm{~g} / \mathrm{mol}$ ) and dimethyl sulfoxide (DMSO) were from Sigma-Aldrich (St. Louis, MO, USA). Methanol was from Merck (Darmstad, Germany). RPMI high glucose medium and supplements (fetal bovine serum, glutamine, penicillin and streptomycin) were from Life Technologies (Grand Island, NY, USA). 


\section{Preparation of PLA nanofiber matrices loaded with SN-38 microcrystals.}

SN-38 microcrystal suspensions were prepared by $\mathrm{pH}$-dependent crystallization the day before the preparation of the nanofiber matrices. The crystallization method takes advantage of the $\mathrm{pH}$-dependent reversible equilibrium between SN-38 carboxylate (water soluble and predominant at basic or neutral pH) and $\mathrm{SN}-38$ lactone (insoluble in water and predominant at acidic $\mathrm{pH}$ ). To form the microcrystals, one volume $(100 \mu \mathrm{L})$ of $\mathrm{SN}-38$ solubilized in basic $\mathrm{pH}(4 \mathrm{mg} / \mathrm{mL}$ in $\mathrm{NaOH} 0.1 \mathrm{~N})$ was mixed with 9 volumes $(900 \mu \mathrm{L})$ of $\mathrm{pH} 5.0$ acetate buffer containing $2 \%$ Pluronic $\AA$ F68. The mixture resulted in a final $\mathrm{pH}$ value of 5.5 and it was stored at $4^{\circ} \mathrm{C}$ for $24 \mathrm{~h}$ with hourly agitation during the first $6 \mathrm{~h}$ to favor the slow precipitation of the SN-38 lactone microcrystals. The size of the crystals at $24 \mathrm{~h}$ was measured by dynamic light scattering (DLS) with a ZetaSizer Nano ZS (Malvern Instruments, Malvern, UK).

SN-38 microcrystal-loaded nanofiber matrices were prepared by electrospinning. PLA (10\% in dichloromethane) was loaded in a $2 \mathrm{~mL}$ syringe and pumped at a constant rate of $0.5 \mathrm{~mL} / \mathrm{h}$ at a $10 \mathrm{kV}$ voltage. The PLA solution was spun for 20 min on a rotating rod wrapped with vegetal paper, to build a first layer of SN-38-free nanofibers that would play the role of rate-controlling membrane and prevent the direct release of the intact drug microcrystals to the physiologic medium. During the following $45 \mathrm{~min}$, the SN-38 microcrystal suspension (loaded in a syringe) was pumped (90 $\mu \mathrm{L} / \mathrm{min})$ simultaneously from the opposite part of the rotating rod, and sprayed with a pneumatic nozzle. The theoretical load of SN-38 in the matrix was $18 \mu \mathrm{g} / \mathrm{cm}^{2}$. Finally, after loading the complete suspension of the drug microcrystals, the PLA solution was spun for extra 20 min to generate another free-drug layer that isolates the cargo. Finally, 
the matrix was dried under vacuum for $24 \mathrm{~h}$, at room temperature. The products (SN-38-loaded nanofiber matrices cut into $0.25,0.5$ or $1 \mathrm{~cm}^{2}$ sheets) were characterized by scanning electron microscopy (SEM; Phenom G1, Eindhoven, The Netherlands), fluorescence microscopy (Leica DM 5000 B, Wetzlar, Germany), and differential scanning calorimetry (DSC 2 STAR $^{\mathrm{e}}$ system simultaneous thermal analyzer with $\operatorname{STAR}^{\mathrm{e}}$ Software V13, Mettler-Toledo, Schwerzenbach, Switzerland) equipped with intra-cooler Huber TC100 under dry $\mathrm{N}_{2}$ atmosphere and In as standard. The amount of SN-38 loaded in the matrices was analyzed by extraction of the drug with methanol and injection of the extract in a high-performance liquid chromatography (HPLC) system with fluorescence detector, as previously described [15].

SN-38 release. Several in vitro and in vivo experiments characterized the release profile of $\mathrm{SN}-38$ from the matrices upon dissolution of internal $\mathrm{SN}-38$ microcrystals in physiologic conditions.

In vitro, SN-38 matrices containing $5 \mu \mathrm{g} \mathrm{SN}-38$ in $0.25 \mathrm{~cm}^{2}(\mathrm{n}=24)$ were placed in glass vials with $5 \mathrm{~mL}$ of pre-warmed PBS $(\mathrm{pH} 7.4)$ and incubated at $37^{\circ} \mathrm{C}$ away from light. At time points $0.25,2,4,8,24,48,72$ and $96 \mathrm{~h}$, three matrices were removed from the vials for drug analysis by HPLC. The removed matrices were vigorously vortexed in $5 \mathrm{~mL}$ methanol to extract the unreleased $\mathrm{SN}-38$ for analysis. The release medium of the remaining matrices was completely replaced with fresh pre-warmed PBS at all sampling times.

We repeated the in vitro release experiment described above though in the presence of the solubilizer HPBCD $(10 \% \mathrm{w} / \mathrm{v}$ in PBS). The sampling times in these experiments were $0.25,0.5,0.75,1,2,4,8$ and $24 \mathrm{~h}$. 
In a third experiment, $0.25 \mathrm{~cm}^{2}$ matrices containing $5 \mu \mathrm{g} \mathrm{SN}-38$ crystals were introduced in 24 well plates containing $400 \mu \mathrm{L}$ of cell culture medium (RPMI supplemented with $10 \%$ FBS, $2 \mathrm{mM} \mathrm{L-glutamine,} \mathrm{penicillin} 100 \mathrm{U} / \mathrm{mL}$ and streptomycin $100 \mu \mathrm{g} / \mathrm{mL}$ ) at $37^{\circ} \mathrm{C}$. We used culture medium to simulate the conditions of the in vitro cytotoxicity studies. The complete volume was removed for HPLC analysis at $8 \mathrm{~h}$ and renewed with fresh medium. At $24 \mathrm{~h}, \mathrm{SN}$ 38 release was analyzed again.

Finally, SN-38 release in vivo was evaluated using $0.5 \mathrm{~cm}^{2}$ matrices containing $9 \mu \mathrm{g}$ of SN-38 and subcutaneously (s.c.) implanted in 12 mice. Mice were sacrificed and matrices were removed at different time points $(1,4,24$ and 48 h). The amount of SN-38 remaining in the matrices was extracted with methanol and analyzed as already described.

Tumor models. Pediatric solid tumor models (neuroblastoma cell lines LAN-1 and SK-N-AS, Ewing sarcoma cell line SK-ES-1 and rhabdomyosarcoma cell line Rh30) were obtained from the repository maintained at Hospital Sant Joan de Déu (HSJD, Barcelona, Spain). Two patient-derived xenografts (PDX), HSJD-NB-005 (neuroblastoma) and HSJD-ES-001 (Ewing sarcoma) were established and maintained in athymic nude mice (Harlan, Barcelona, Spain) as previously described $[15,22]$. Primary cultures were established by disaggregation of the PDX models with collagenase-DNase enzymes (SigmaAldrich). Additional information of the PDX models is available in Table 1 and published elsewhere [23]. The research performed with mice was approved by the institutional ethics committee. 
In vitro activity. The MTS assay (Promega, Fitchburg, WI, USA) was used for determination of cell viability after in vitro cytotoxicity experiments. Each treatment condition was assayed at least in triplicate.

To determine the activity of SN-38 against the pediatric solid tumor cell models (LAN-1, SK-N-AS, SK-ES-1 and Rh30 cell lines; HSJD-NB-005 and HSJD-ES001 primary cultures), we performed assays in 96 well-plates with 3000 cells per well and 3-4 days of incubation with the drug (stock solubilized in DMSO), as previously described [24].

The activity of the SN-38 matrices $\left(0.25 \mathrm{~cm}^{2} ; 5 \mu \mathrm{g} \mathrm{SN}-38\right)$ was studied in 24well plates. For this, 12,000 cells were plated in each well and cultured until they formed monolayers of tumor cells covering the growth area of $1.9 \mathrm{~cm}^{2}$ provided by the well. In a first experiment, SN-38 matrices were added to the culture monolayers and removed after $8,24,48$ and $96 \mathrm{~h}$, renewing the culture medium at each time point. After $96 \mathrm{~h}$, cell viability was determined as described before. Blank matrices (no SN-38 content) were used as a control.

In a second experiment, $\mathrm{SN}-38$ matrices were preconditioned in cell culture medium without cells $(400 \mu \mathrm{L})$ at $37^{\circ} \mathrm{C}$ for 24 or $48 \mathrm{~h}$. After the preconditioning stage, the matrices were transferred to the wells with cell monolayers in culture. Thus, a significant fraction of the drug would have been released before cell treatments. After $72 \mathrm{~h}$ of incubation, the matrices were removed and the viability of the cells was determined.

In a third assay, blank matrices and SN-38-loaded matrices were co-incubated for $24 \mathrm{~h}$ in 24 -well plates containing $400 \mu \mathrm{L}$ cell-free culture medium. Thus, SN38 from drug-loaded matrices that underwent solubilization in the medium could 
be absorbed by blank matrices during the co-incubation time. Then, all the matrices were washed by fast immersion in cold PBS, and transferred to 24 well-plates containing tumor cells monolayers in culture. Cell viability was determined after $72 \mathrm{~h}$.

Systemic pharmacokinetics. One $\mathrm{cm}^{2} \mathrm{SN}-38$-loaded matrix containing $18 \mu \mathrm{g}$ SN-38 was implanted S.c. in 6-weeks old athymic nude mice $(n=13)$. Such administration provides a $1 \mathrm{mg} / \mathrm{kg} \mathrm{SN}-38$ dose (the average weight of the mice was $18 \mathrm{~g}$ ). At $0.25,0.5,1,3,6,12,24$ and $48 \mathrm{~h}$ after matrix implantation, mice were bled $(50 \mu \mathrm{L})$ by the retroorbital plexus.

A second group of mice received an intravenous injection of irinotecan at an equimolar dose $(44 \mu \mathrm{g}$ irinotecan trihydrate in $100 \mu \mathrm{L}$ vehicle) and they were bled at $0.25,1,4$ and $10 \mathrm{~h}$ after injection. To calculate the equimolar dose of irinotecan and SN-38, we considered published work in which $70 \%$ of systemic irinotecan is converted to $\mathrm{SN}-38$ in nude mice [18].

A maximum of 3 blood samples were obtained from each mouse. SN-38 in plasma was analyzed as previously described [15]. We used the trapezoid method to calculate the area under the concentration-time curve (AUC) of SN38 lactone in plasma.

Local pharmacokinetics of SN-38 matrices in the surgical bed. To study the release of soluble SN-38 in the virtual space of the subcutaneous surgical bed upon administration of $\mathrm{SN}-38$ matrices or soluble prodrug irinotecan, we performed a series of in vivo microdialysis experiments in 9 nude mice, as previously described [15]. First, we inserted a CMA 20 microdialysis probe (CMA, Kista, Sweden) s.c. in the flank of the mouse, under isoflurane 
anesthesia. The probe was infused continuously with perfusate (PBS containing $10 \%$ HPBCD) using an infusion pump at $0.5 \mu \mathrm{L} / \mathrm{min}$. The microdialysis probe was stabilized for $1 \mathrm{~h}$ before the administration of the dose. A first group of mice $(n=3)$ was anesthetized with isoflurane, a small incision was made in the skin 1 $\mathrm{cm}$ away from the probe, and a $1 \mathrm{~cm}^{2} \mathrm{SN}-38$ matrix $(18 \mu \mathrm{g} \mathrm{SN}-38 ; 1 \mathrm{mg} / \mathrm{kg})$ was inserted between the probe and the mouse skin. The wound was sutured with clips. A second group $(n=3)$ was slightly anesthetized with isoflurane to receive a local injection of irinotecan s.c. in the probe area, at a dose $(44 \mu \mathrm{g}$ irinotecan in $100 \mu \mathrm{L}$ ) that was equimolar to the one used for SN-38. A third group $(n=3)$ received $44 \mu$ irinotecan intravenously. Dialysate samples were collected in an autosampler and analyzed by HPLC [15]. Probes were calibrated using the mean recovery value (70\%) previously determined in vivo at steady state $\mathrm{SN}-38$ plasma levels [15]. Blood samples $(30 \mu \mathrm{L})$ were obtained at $0.25,1$, 4 and $24 \mathrm{~h}$ after dose administration and every $24 \mathrm{~h}$ until the end of the experiment with SN-38 matrices; and at $0.25,1,4$ and $10 \mathrm{~h}$ after irinotecan injections. After HPLC analysis, we calculated the AUC of SN-38 lactone in the surgical bed and plasma from each individual microdialysis experiment.

\section{Diffusion of locally released SN-38 through the solid tumor tissue. To} investigate the depth of local SN-38 diffusion into the extracellular fluid (ECF) of tumor tissue upon localized release in the tumor periphery by $\mathrm{SN}-38$ matrices, we implanted (s.c.) the HSJD-NB-005 PDX model in one flank of 16 mice. When the tumor reached a diameter of at least $10 \mathrm{~mm}$, we anesthetized the mice with isoflurane and inserted a microdialysis probe into the tumor. The 4 $\mathrm{mm}$ length probe was introduced so that it was aligned in parallel to the tumor 
surface, at a determined distance from the tumor surface (range 0-8 $\mathrm{mm}$, depending on each individual experiment).

After probe equilibration, a small incision was made in the skin $1 \mathrm{~cm}$ away from the probe, and a $1 \mathrm{~cm}^{2} \mathrm{SN}$-38-loaded matrix (18 $\mu \mathrm{g} \mathrm{SN-38;1} \mathrm{mg} / \mathrm{kg}$ ) was inserted in 14 mice between the probe and the mouse skin. In a subgroup of 2 mice, the SN-38 matrix was implanted in the contralateral flank. The wound was sutured with clips. Dialysate samples were taken overnight. At the endpoint of the experiment the probe was perfused with methylene blue to stain the probe track, the tumor was sectioned transversally to the probe track and the distance between the blue track and the SN-38 matrix was measured with a caliper. After HPLC analysis, we calculated the AUC (0-9 h) of SN-38 lactone in tumor ECF from each individual microdialysis experiment.

In vivo antitumor activity. We evaluated the local activity of the SN-38 matrices in the PDX models HSJD-NB-005 and HSJD-ES-001. In a first set of experiments, both tumor models were implanted s.c. in both flanks of $n=8$ and $\mathrm{n}=7$ mice, respectively. When the tumors reached $1000-2000 \mathrm{~mm}^{3}$, we performed a subtotal resection surgery under ketamine-xylazine anesthesia. At that point, tumors were infiltrating the surrounding tissues (muscle and skin). Most of the tumor volume was macroscopically removed from both flanks with the exception of a well-vascularized and viable tumor fragment of approximately $2 \times 1 \mathrm{~mm}$ (length $\mathrm{x}$ width). We covered such tumor rest with one SN-38 matrix in one flank, and a blank matrix in the opposite. Wounds were closed with clips and the animals recovered. The size of tumor recurrences in both flanks was measured with a caliper at different time points during the following 3 weeks. 
Finally, we run a survival experiment in the HSJD-NB-005 PDX model. For the survival studies, 31 nude mice bearing s.c. tumors of $0.1-0.5 \mathrm{~cm}^{3}$ in one flank were randomized in 4 groups of 7-8 mice for subtotal tumor resection surgery. One group received local SN-38 matrix (18 $\mu \mathrm{g} \mathrm{SN}-38)$; a second group was treated with equimolar local s.c. irinotecan; a third group received equimolar systemic irinotecan (via intraperitoneal injection); and a fourth group received a blank matrix on the tumor bed. Tumor recurrences were measured 3 times a week and mice were sacrificed when the tumor diameter reached $2 \mathrm{~cm}^{3}$. The study finalized at day 100 post-resection surgery. Animal survival was defined as the time interval between the initial date of treatment and the date in which 2 $\mathrm{cm}^{3}$ tumor volume was reached.

Statistics. Statistical analysis was performed with Graphpad Prism 5 software (La Jolla, CA). Aggregate data are presented as mean \pm SD. Pharmacokinetic data was log-transformed before analysis and the one-way ANOVA test with Bonferroni's multiple comparison was used to compare parameters between groups. Paired t test was used to compare tumor size in treated animals with tumors in both flanks (treated versus control). Median survivals were calculated using Kaplan-Meier curves and the log-rank test with Bonferroni-corrected threshold was applied for comparison of multiple survival curves.

\section{Results}

Characterization of SN-38-loaded matrices. The size of the SN-38 microcrystals before spraying in the nanofiber matrices was $1.7 \pm 0.34 \mu \mathrm{m}$ (mean \pm SD z-average; poly-dispersion index 0.201). SEM analysis of dry 
matrices showed the presence of SN-38 microcrystals on the surface of PLA nanofibers, covered by a thin layer of surfactant (Figure 1A). The presence of SN-38 microcrystals in the internal layer of the matrix (Figure 1B) but not in the external layer (Figure 1C) was also detected by fluorescence microscopy.

DSC analysis provided comparable thermograms of blank matrices and SN-38loaded matrices that included characteristic thermal transitions of semicrystalline PLA, with the exception of an endothermal peak at $232{ }^{\circ} \mathrm{C}$ in the latter that was consistent with the presence of crystalline SN-38 (Figure 1D). Drug load was analyzed in $1 \mathrm{~cm}^{2} \mathrm{SN}-38$ matrices containing a theoretical load of $18 \mu \mathrm{g} \mathrm{SN}-38 / \mathrm{cm}^{2}$. Loading efficiency was $97.7 \pm 9.2 \%($ mean $\pm \mathrm{SD} ; \mathrm{n}=9)$.

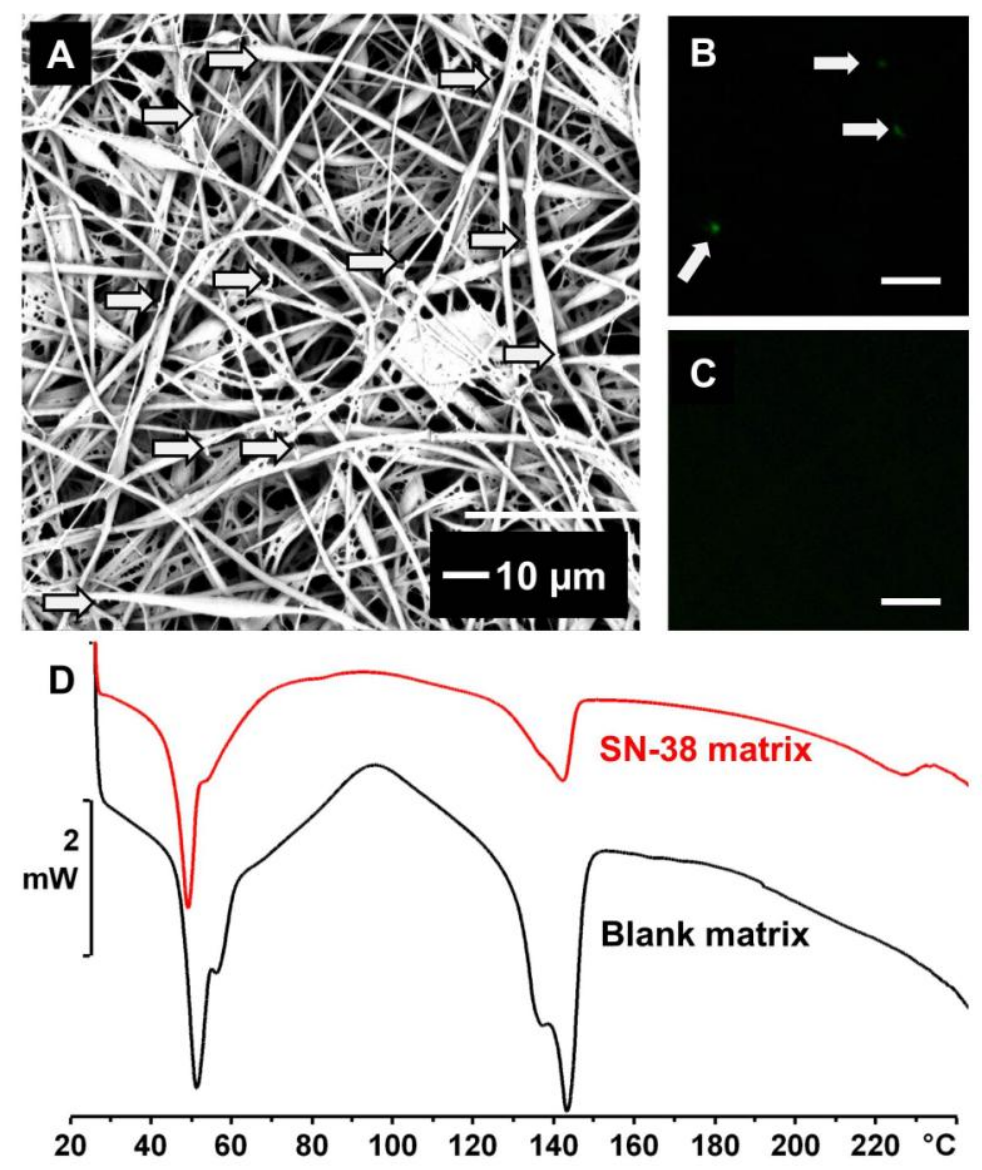

Figure 1. Physical characterization of the SN-38-loaded matrices. A. SEM micrograph of the internal layer of the nanofiber matrix. Note the fibers are covered by a layer of 
surfactant (Pluronic F68). SN-38 microcrystals are labeled with arrows. B. Fluorescent image of SN-38 microcrystals in the internal layer of the matrix. Bar $=10 \mu \mathrm{m}$. C. Absence of fluorescent SN-38 microcrystals in the external layer of the matrix. Bar $=10$ $\mu \mathrm{m}$. D. DSC thermograms of SN-38-loaded and SN-38 free (blank) matrices. The endothermal peak at $232{ }^{\circ} \mathrm{C}$ in the $\mathrm{SN}$-38-loaded matrices corresponds to crystalline SN38.

SN-38 release from matrices. Figure $2 \mathrm{~A}$ shows the in vitro release profile from SN-38-loaded matrices incubated in the absence of HPBCD solubilizer. Release was sustained in time and achieved $72.1 \pm 10.6 \%$ of the payload after $96 \mathrm{~h}$ of incubation. Unreleased SN-38 remained mainly in the lactone form (98.5 $\pm 0.2 \%)$ and not in the carboxylate one $(1.5 \pm 0.2 \%)$. Drug analysis was not performed in the release medium because, in the absence of HPBCD, SN-38 extensively binds to glassware and plasticware, leading to irreproducible results [15]. As expected, the addition of HPBCD permitted reproducible drug analysis in the release medium in a cumulative release experiment (Figure 2B). Due to the presence of HPBCD in the release medium, the released SN-38 was watersolubilized and the matrices were completely deprived of the cargo within $24 \mathrm{~h}$; drug load in the matrices was $0.49 \pm 0.77 \%$ at the end of the study. In vivo release data from individual $\mathrm{SN}-38$ matrices overlapped the in vitro release curve (Figure 2A). SN-38 remaining in the matrices of the in vivo assay was mainly lactone $(93.1 \pm 3.2 \%)$ over carboxylate SN-38 $(6.9 \pm 3.2 \%)$ at all the time points. In culture medium, SN-38 matrices achieved concentrations above $1 \mu \mathrm{M}$ (range $0.88-6.07 ; \mathrm{n}=6$ ) after $8 \mathrm{~h}$ of incubation, and above $0.1 \mu \mathrm{M}$ at $24 \mathrm{~h}$ (range 0.14-3.54; $n=6$ ) (Figure 2C). 


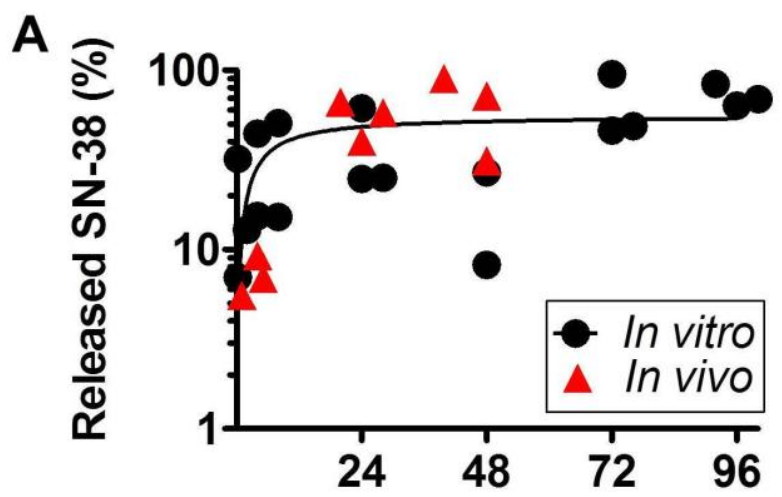

B

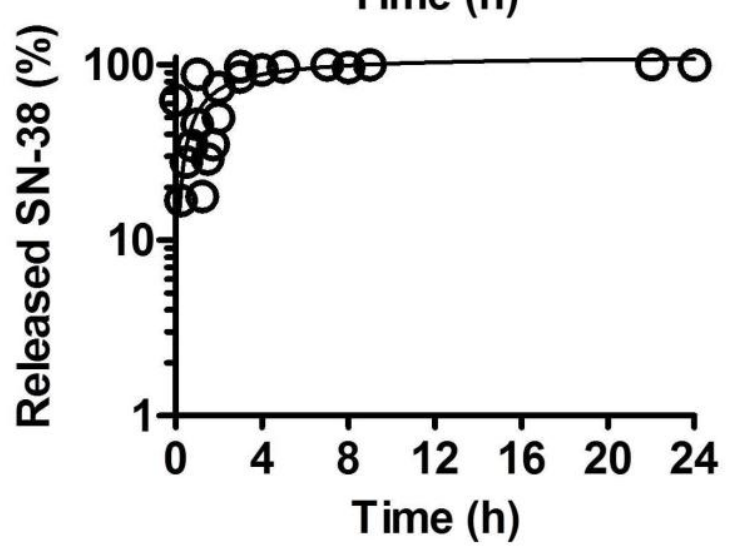

C

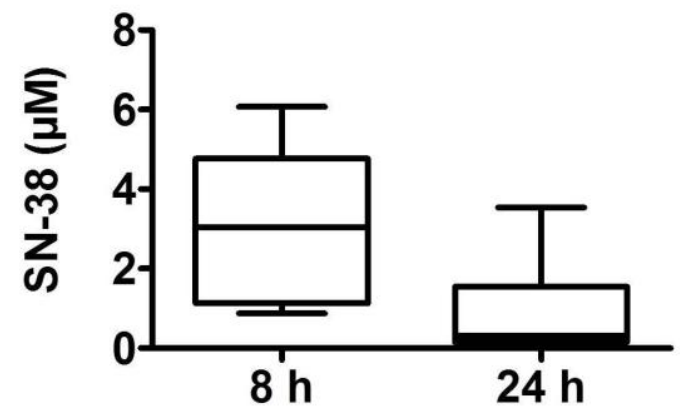

Figure 2. Release from the $\mathrm{SN}-38$ matrices. A. In vitro release profile without solubilizer. The remaining amount of $\mathrm{SN}-38$ was analyzed in individual matrices $(\mathrm{n}=$ 24; black dots). In vivo release data (red dots) are superposed in the in vitro curve. Each dot represents one individual matrix removed from one mouse $(n=12)$. B. Cumulative in vitro release profile from SN-38 matrices in the presence of HPBCD in the release medium $(n=3)$. Values were corrected to $100 \%$ upon analysis of the remaining amount of $\mathrm{SN}-38$ in the matrices at the end of the experiment. C. Concentrations achieved in cell culture medium $(400 \mu \mathrm{L})$ upon incubation of SN-38 matrices containing $5 \mu \mathrm{g} \mathrm{SN}-38$ during 8 or 24 hours $(n=6)$. 
In vitro activity. As shown in Table 2, all the pediatric solid tumor cell models

A

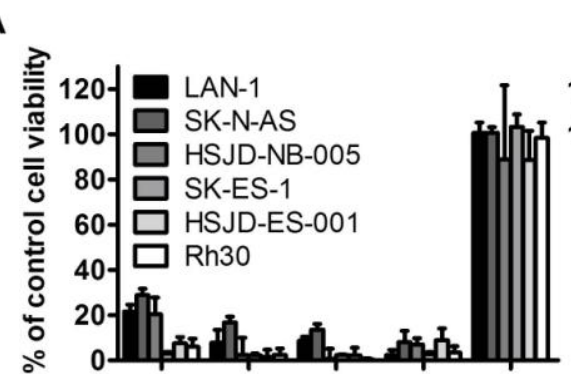

B

(Figure 3A). SN-38-loaded matrices preconditioned in cell culture medium during 24 or $48 \mathrm{~h}$ conserved their antiproliferative activity when transferred to cell monolayers in culture (Figure 3B). Blank matrices co-cultured with $\mathrm{SN}-38$ matrices and transferred subsequently to cell cultures did not acquire antiproliferative activity (Figure 3C).

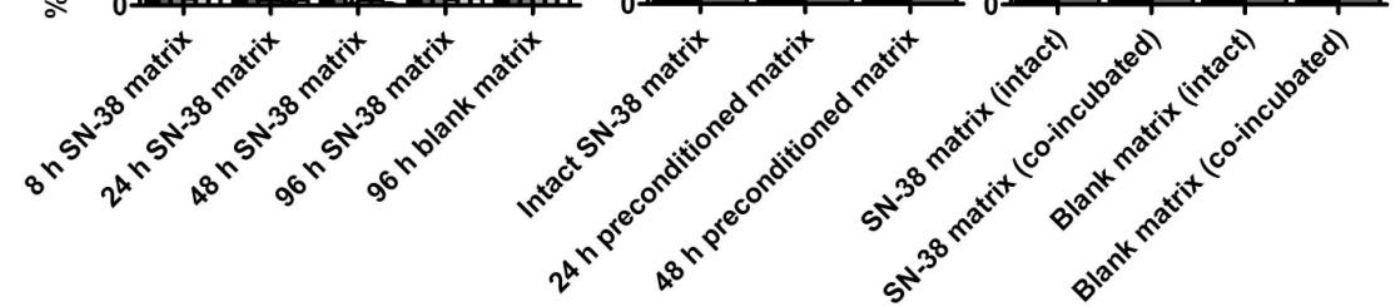

Figure 3. Antiproliferative activity of the SN-38 matrices. A) Activity of the SN-38 matrices against pediatric cell culture monolayers upon exposures ranging 8-96 $\mathrm{h}$. Blank matrices did not exhibit antiproliferative activity. B) SN-38 matrices preconditioned in cell culture medium during 24 or $48 \mathrm{~h}$ conserved their activity, as compared to the activity of the not preconditioned (intact) formulation. C) Blank matrices co-incubated with SN-38 matrices during $24 \mathrm{~h}$ did not acquire antiproliferative activity, whereas co-cultured $\mathrm{SN}-38$ matrices conserved their activity as compared to the activity of the intact SN-38 matrices. All the experiments were performed in triplicate. 
Plasma pharmacokinetics. SN-38 lactone levels in mouse plasma are shown in Figure 4. Mice receiving SN-38 matrices achieved median lactone SN-38 peak plasma level $\left(C_{\max }\right)$ of $1.05 \mathrm{ng} / \mathrm{mL}$ (range $0.53-1.27$ ) at $1 \mathrm{~h}$ after s.c. insertion. SN-38 plasma concentrations were below the limit of quantification $(0.25 \mathrm{ng} / \mathrm{mL})$ at $12 \mathrm{~h}$. The calculated AUC $(0-6 \mathrm{~h})$ was $3.78 \mathrm{ng}{ }^{*} \mathrm{~h} / \mathrm{mL}$. After intravenous injections, SN-38 plasma levels were fitted to a two compartment model and the extrapolated $\mathrm{C}_{\max }$ was $122 \mathrm{ng} / \mathrm{mL}$ and the calculated AUC (0-7 h) was $104 \mathrm{ng}^{*} \mathrm{~h} / \mathrm{mL}$.

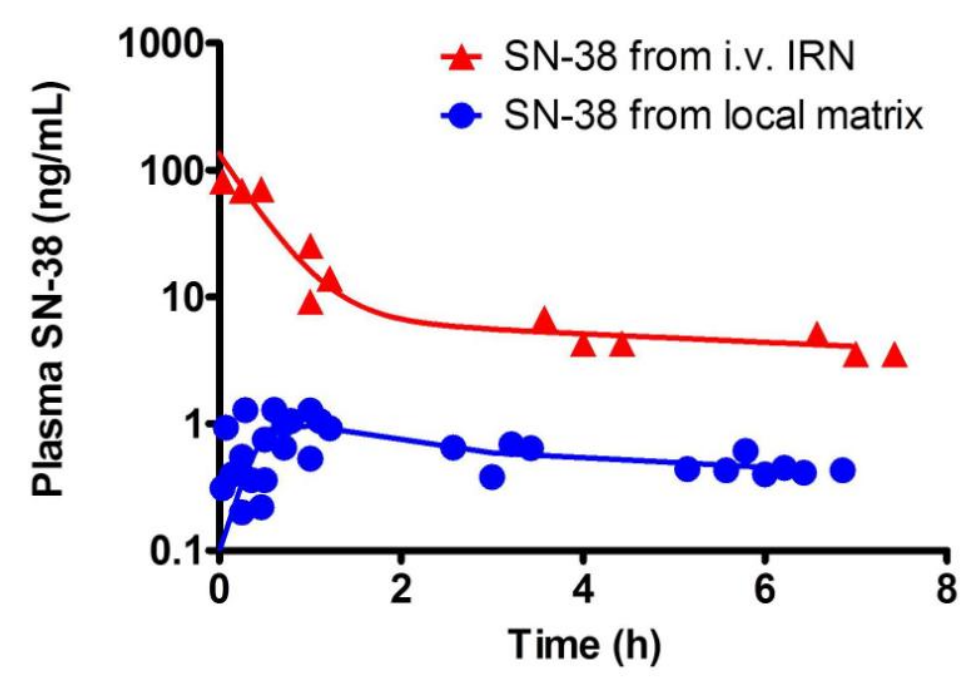

Figure 4. Plasma concentration-time data of SN-38 lactone upon administration of equimolar dosages of $\mathrm{SN}-38$ matrices (1 mg/kg; s.c.; local matrix) and systemic irinotecan (i.v. IRN) in nude mice ( $n=13$ and $n=4$, respectively).

SN-38 pharmacokinetics in the surgical bed. Lactone SN-38 concentrations were quantified in the s.c. space and plasma from mice with microdialysis probes placed in the s.c. surgical bed (Figure 5). SN-38-loaded matrices induced high drug concentrations in the surgical bed $(408 \pm 284 \mathrm{ng} / \mathrm{mL}$; mean concentration during the first $12 \mathrm{~h}$ of three experiments). Such levels were sustained and above $10 \mathrm{ng} / \mathrm{mL}$ (i.e. $25 \mathrm{nM}$; above the IC50 in 4 out of 6 cell 
models shown in Table 2) until the end of the 4-day microdialysis experiments. A representative experiment in which the probe was in close proximity to the SN-38 matrix is shown in Figure 5A. In comparison, local s.c. injection of equimolar irinotecan achieved peak levels in the surgical bed of $41.6 \pm 3.4$ $\mathrm{ng} / \mathrm{mL}$ (mean $\mathrm{C}_{\max }$ from 3 experiments), which dropped to less than $10 \mathrm{ng} / \mathrm{mL}$ after $7 \mathrm{~h}$ (Figure 5B). Maximum SN-38 levels in the surgical bed of mice injected i.v. were $3.55 \pm 1.16 \mathrm{ng} / \mathrm{mL}$ and they were below $0.25 \mathrm{ng} / \mathrm{mL}$, the limit of quantification (L.O.Q.), after $6 \mathrm{~h}$ (Figure 5C).
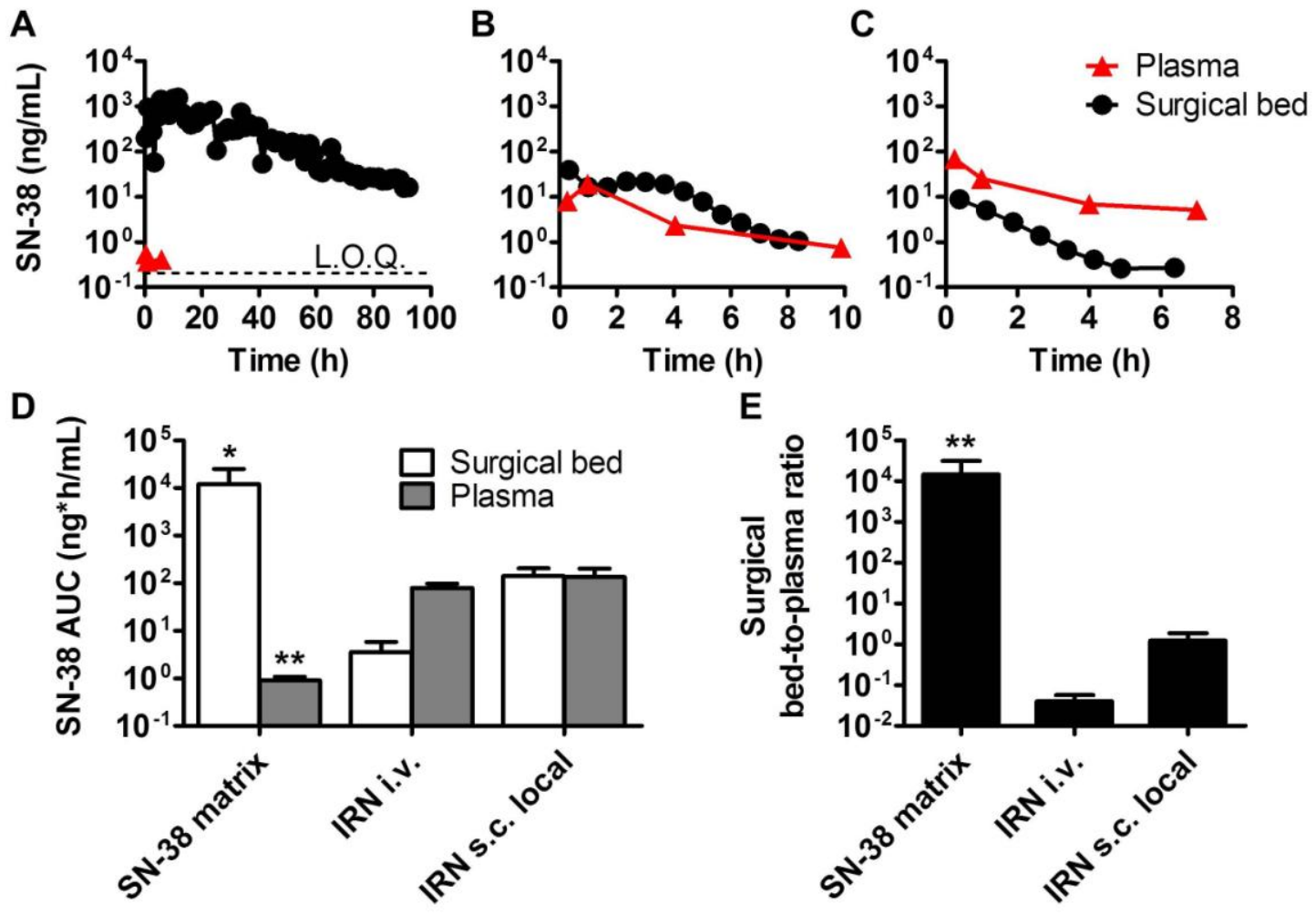

Figure 5. SN-38 pharmacokinetics in the s.c. surgical bed and in plasma upon administration of equimolar doses from s.c. SN-38 matrices $(1 \mathrm{mg} / \mathrm{kg})$ or irinotecan (IRN), either s.c. or i.v. Representative individual experiments are shown in A (SN-38 matrix, s.c.), B (IRN s.c. local), and C (IRN i.v.). Black dots represent SN-38 levels (dialyzable fraction, recovery-corrected) in the virtual space of the s.c. surgical bed. Red triangles are plasma data. L.O.Q: Limit of quantification $(0.25 \mathrm{ng} / \mathrm{mL})$. D. SN-38 AUCs in surgical bed and plasma. E. Surgical bed-to-plasma SN-38 AUC ratios. Data are mean $\pm S D$ from triplicate experiments. ${ }^{*} P<0.01$ and ${ }^{* *} P<0.001$ as compared to 
IRN i.v. and IRN s.c. local groups (one-way ANOVA test with Bonferroni's multiple comparison).

SN-38 plasma levels were slightly above the L.O.Q. only until $6 \mathrm{~h}$ in mice receiving SN-38 matrices, as compared to higher plasma levels in mice receiving irinotecan s.c. or i.v. (Figure 5A-C). SN-38 exposure (AUC) in the surgical bed surrounding the SN-38-loaded matrices, and surgical bed-toplasma AUC ratio, were at least 2 logs higher as compared to the exposures of animals receiving s.c. or i.v. irinotecan (Figures 5D and 5E). Concomitantly, plasma AUC in animals receiving SN-38-loaded matrices was lower than in counterparts receiving irinotecan s.c. or i.v (Figure 5D).

Diffusion of locally released SN-38 through the solid tumor tissue. Upon measuring the distance between the peritumoral SN-38-loaded matrix and the intratumoral microdialysis probe track at the experimental endpoint, we grouped the experiments in distance ranges of $0-1 \mathrm{~mm}(\mathrm{n}=4), 1-2 \mathrm{~mm}(\mathrm{n}=3), 2-5 \mathrm{~mm}$ $(\mathrm{n}=4)$, and 5-10 $\mathrm{mm}(\mathrm{n}=3)$. Release from $\mathrm{SN}$-38-loaded matrices resulted in significantly higher SN-38 concentrations in tumor ECF when the probe was placed up to $2 \mathrm{~mm}$ distance from the matrix, as compared to when such distance was $>2-5 \mathrm{~mm}(\mathrm{P}<0.05$; Figure $6 \mathrm{~A})$. Mean $\mathrm{SN}-38$ concentration achieved in the $2-5 \mathrm{~mm}$ distance range experiments was $2.0 \pm 1.1 \mathrm{ng} / \mathrm{mL}$ (i.e., $5.1 \pm 2.8 \mathrm{nM}$ ) and thus it was below the IC50 of 4 out of 6 cell models studied in this work (Table 2). SN-38 in tumor ECF was undetectable at distances greater than $5 \mathrm{~mm}$ or when it was released in the flank contralateral to the tumor. Tumor SN-38 exposures (AUC 0-9 h) were significantly higher in the $<1 \mathrm{~mm}$ and 1-2 mm distance groups, as compared to the $2-5 \mathrm{~mm}$ group $(P<0.01$ and $P<0.05$; Figure 6B). Exposures were below the detection limit when the 
distance between tumor and probe was greater than $5 \mathrm{~mm}$. Two representative microdialysis experiments are shown from mice with s.c. probes located either in close contact with the matrix (Figure $6 \mathrm{C}$; $<1 \mathrm{~mm}$ ) or distant from the matrix (Figure 6D; $4 \mathrm{~mm}$ ).

A
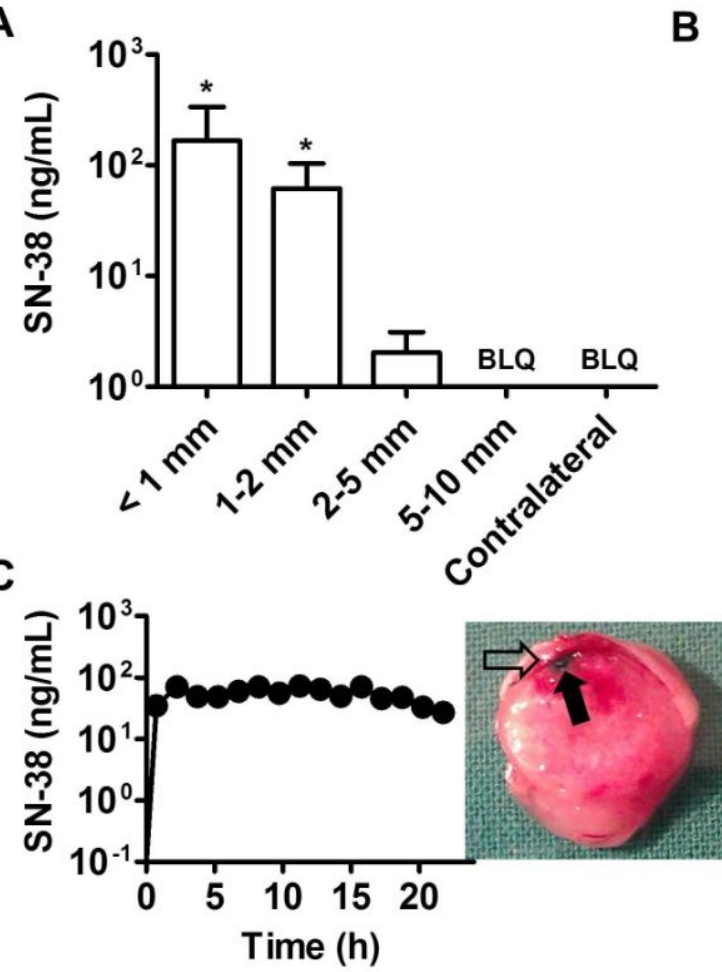

B

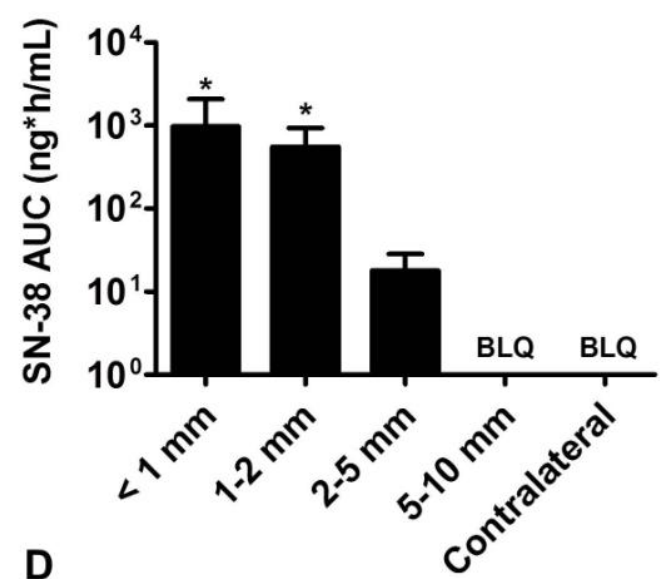

D

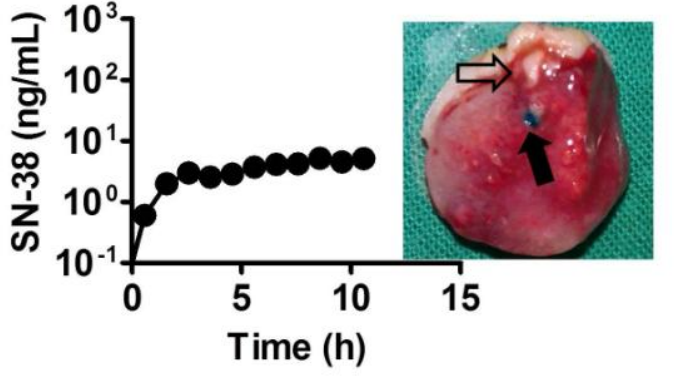

Figure 6. Intratumor penetration of $\mathrm{SN}-38$ upon administration of $\mathrm{SN}-38$ matrices. A. SN-38 concentrations in tumor ECF sampled by an intratumoral microdialysis probe positioned at different depths $(<1 \mathrm{~mm}, 1-2 \mathrm{~mm}, 2-5 \mathrm{~mm}, 5-10 \mathrm{~mm}$ ) relative to the position of the SN-38 matrix covering the tumor periphery. Mean \pm SD concentrations during the first $9 \mathrm{~h}$ of 3-4 experiments are shown. "Contralateral" refers to experiments in which the SN-38 matrix was located s.c. in the flank contralateral to the intratumoral probe ( $n=2$ experiments). B. AUC data obtained in each relative position (mean $\pm S D$ ). C, D. Individual microdialysis experiments showing plots of SN-38 levels in tumor ECF. Side pictures in $C$ and $D$ are corresponding tumor sections displaying the relative position between the probe track (black arrow) and the SN-38 matrix (empty arrow). The distance track-matrix was $<1 \mathrm{~mm}$ in $C$ and $4.3 \mathrm{~mm}$ in $\mathrm{D}$. BLQ: Below limit of quantification. ${ }^{*} \mathrm{P}<0.05$ as compared to the $2-5 \mathrm{~mm}$ group (one-way ANOVA test with Bonferroni's multiple comparison). 
In vivo antitumor activity. SN-38 matrices delayed tumor growth in the surgical bed after subtotal resection of PDX models HSJD-NB-005 and HSJDES-001. Contralateral tumors receiving blank matrices were significantly larger than the treated ones at the studied time points $(P<0.05$; paired $t$ test; Figure 7).

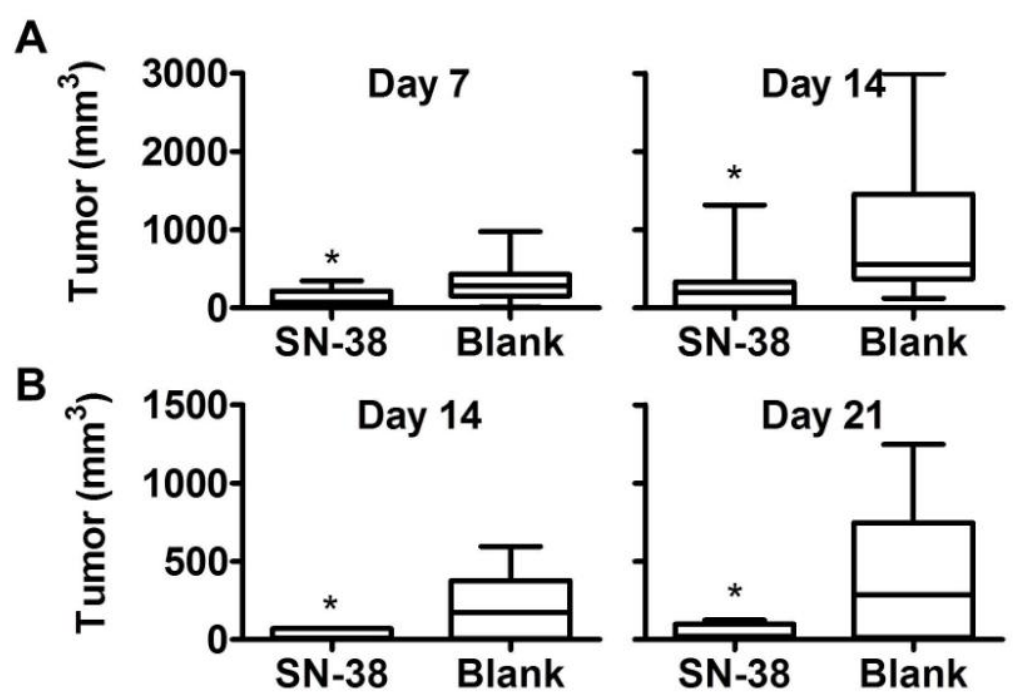

Figure 7. In vivo activity of the SN-38 matrices in HSJD-NB-005 (A) and HSJD-ES-001 (B) PDX models after subtotal bilateral tumor resection. Time from surgery until recurrence in the tumor side treated with $\mathrm{SN}-38$ matrices (SN-38) was significantly delayed as compared to recurrence in the opposite flank treated with blank matrix (Blank). Mean and SD data from 8-11 mice with bilateral tumors are represented. ${ }^{*} \mathrm{P}<$ 0.05 (Paired $t$ test). Sampling times are different between both models because of different tumor growth kinetics.

Survival. Median survival of the animals receiving SN-38-loaded matrices $(n=$ 8), blank matrices $(n=8)$, s.c. irinotecan $(n=7)$ and systemic irinotecan $(n=8)$ after subtotal tumor resection was 76, 37, 46 and 40 days, respectively (Figure 8). Mice treated with $\mathrm{SN}$-38-loaded matrices after surgery survived significantly longer than mice treated with blank matrices $(P=0.0068)$. In contrast, localized 
s.c. or systemic irinotecan did not provide a significant benefit in animal survival ( $P=0.3292$ and $P=0.5944$, respectively $)$ as compared to blank matrices. Treatment with $\mathrm{SN}-38$ matrices performed better than s.c. irinotecan $(\mathrm{P}=$ 0.0080). Difference in survival between the $\mathrm{SN}$-38-loaded matrix group and the systemic irinotecan group was not statistically significant $(P=0.120)$.

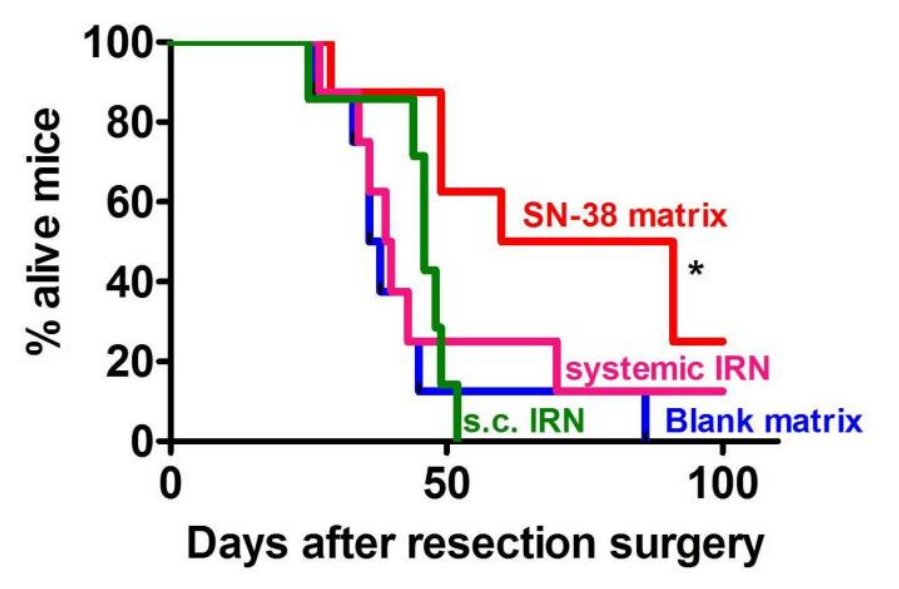

Figure 8. Survival of mice bearing HSJD-NB-005 tumors, upon subtotal resection and treatment with local SN-38 matrices (SN-38 matrix) or control treatments (Blank matrix, systemic IRN, s.c. IRN). Median survival of each group was determined and the Bonferroni-corrected threshold was applied for comparison of multiple survival curves (i.e. for 4 groups, there are 6 pairwise comparisons). With the Bonferroni correction, $P$ $<0.0083$ was considered significant. ${ }^{*} \mathrm{P}=0.0080$ as compared to control group (Blank matrix).

\section{Discussion}

Local tumor control in pediatric oncology requires new treatments in addition to surgery, to delay or replace radiotherapy. However, such innovative treatments are currently unavailable in the clinic. Motivated by this unmet need, we have developed a clinically translatable nano-DDS for the local release of SN-38, a poorly-water soluble camptothecin with proved antitumor activity against several pediatric solid tumors. To select the surgical scenarios in which the new system 
would be suitable, we first conducted preclinical experiments to characterize the microdialysis sampling in vivo. We observed that the released drug achieves high concentrations in the virtual space of the surgical bed and it penetrates a maximum distance of $2 \mathrm{~mm}$ within the bulk of the tumor. Therefore, we propose that surgeries achieving macroscopically complete resection or minimal tumor residues surrounding vital structures could be optimal candidates for the new treatment strategy. Subsequently, we developed a model of subtotal tumor resection in clinically relevant pediatric PDX models and we used such model to provide evidence of the activity of the SN-38 DDS to inhibit tumor growth after surgical resection.

Irinotecan is potently active as single agent in preclinical models of neuroblastoma [17], rhabdomyosarcoma [25] and Ewing sarcoma (own results in PDX models, unpublished). In our present study, we confirmed the potency of its active metabolite $\mathrm{SN}-38$ against pediatric cancer cell lines and against primary cultures derived from PDX models established at our institution (Tables 1,2 and Figure 3). However, irinotecan in pediatric cancer clinical trials has not replicated the reported preclinical activity, either as single agent $[26,27]$ or combined with other drugs [28]. The reasons for this lack of correlation between preclinical and clinical efficacies are not totally understood, but they could be related to suboptimal SN-38 distribution in human tumors. Greater activity of murine carboxylesterases (the enzymes required to metabolize systemic irinotecan into $\mathrm{SN}-38$ ) as compared to the human counterparts leads to $70 \%$ irinotecan conversion into $\mathrm{SN}-38$ in mice as opposed to less than $10 \%$ in humans $[18,19,29,30]$. As a consequence of this, higher tumor exposure to 
SN-38 might be achieved in the murine model. Further evidence supports that increased SN-38 exposure in tumors leads to improved survival of mice with aggressive neuroblastoma [31].

The identification of the pharmacokinetic limitations of irinotecan warranted the development of several DDS carrying $\mathrm{SN}-38$ for systemic and local administration [32, 33]. In our study, an electrospun nanofiber matrix made of an approved biodegradable biocompatible polyester, PLA, was chosen as drug carrier because the shape and malleability of the matrix is adaptable to the surgical bed that may vary between patients. The manufacturing process (simultaneous microcrystal spraying during polymer electrospinning) was selected after a series of preliminary feasibility studies where the drug was blended with the polymer solution and electrospun, an approach that resulted in extremely slow delivery rates controlled mainly by the polymer degradation (data not shown and [33]). The final configuration took advantage of the poor solubility and dissolution rate of $\mathrm{SN}-38$ in the physiologic medium that was slow enough to produce a bimodal release kinetics, as demonstrated in vitro and in vivo (Figure 2A,B). This type of release is likely clinically relevant because initially faster release would achieve high local drug concentration (as we demonstrate by the microdialysis sampling approach), whereas long-lasting release at a slower rate could be important for recruiting more tumor cells at the S-phase of the cycle. Because the microcrystals are incrusted in the internal layers of the nanofiber matrix, the drug cannot be released from the DDS unless the fluids permeating through the highly porous nanofiber mesh solubilize it.

To demonstrate that the drug crystals loaded in the polymeric matrix provide a sustained localized release and confer long-term antitumor activity, we 
performed two specifically designed in vitro experiments (preconditioning in culture medium and co-incubation with blank matrices). In fact, preservation of long term activity might be related to the chemical stabilization of the SN-38 lactone in the hydrophobic PLA matrix, in line with previous studies reporting that campthothecins loaded in polymers or gels were stabilized in the active lactone form [34-37]. Drugs blended in polymers are usually in molecular dispersion (amorphous) and not crystalline [38]. Our work shows for the first time that microcrystals of a poorly water-soluble hydrolysable drug such as SN38 could be stabilized by incorporation into the nanofiber mesh.

One fundamental contribution of our research in pediatric cancer is the application of the microdialysis method to study local drug delivery to the tumor bulk in vivo. In this work, we showed that the local penetration was restricted to $2 \mathrm{~mm}$ depth into the solid tumor tissue. These findings are consistent with the report of Arifin et al. using computer simulation that showed penetration of less than $2 \mathrm{~mm}$ after local (intracerebral) BCNU release from Gliadel implants [14]. Interestingly, the Arifin study defined the term "therapeutic penetration" as "the length from the remnant tumor interface for which the drug concentration is above the therapeutic concentration" [14]. Other authors have used terminal sampling methods to propose that localized drug delivery might achieve such therapeutic penetration to ratios greater than $2 \mathrm{~mm}$ [38]. However, we suggest that the terminal sampling methods (after animal death) might have overestimated the drug penetration distance due to maintained drug release from the DDS into the tissue until sample collection. For instance, in a previous study, we demonstrated that upon local administration in the periocular space of topotecan, another camptothecin used in the treatment of pediatric solid tumors 
like retinoblastoma, postmortem sampling dramatically increased topotecan levels in the posterior segment of the eye (an organ with positive pressure, like most solid tumors). The reason for this observation would be the termination of the active drug elimination process upon the animal death [39]. In another study of local drug release in ocular cancer, we increased intraocular drug distribution by the administration of concomitant local adrenaline to inhibit local blood flow [40]. We thus speculate that the inclusion of vasoconstrictors in the local DDS might help achieve deeper therapeutic drug penetration in solid tumors and further studies will be focused on this topic.

Previous preclinical models of local DDS activity against extracranial tumors have been based on the insertion of the local DDS next to intact solid tumors established in mice, either in s.c. or orthotopic location. In such scenario, local DDS releasing paclitaxel and cisplatin have performed poorly to control s.c. glioma xenografts [41] and mouse uterine tumors [42], respectively. Such results could be explained by our studies of therapeutic penetration into the solid tumor bulk. In contrast, other preclinical models reproduced the context of "microscopic tumor rests", either by the injection of cancer cells on top of a previously implanted local DDS [37] or by the total macroscopic resection of established xenografts previous to the insertion of the local DDS [43-45]. Such strategies have been used in the work of Grinstaff and Colson to study the activity of hydroxycampthothecin-loaded DDS polymer films against lung carcinoma cells [37] and paclitaxel-loaded films against non-small-cell lung cancer cell line xenografts [44] and sarcoma xenografts [43], achieving prolonged control of local tumor recurrences. 
The results of our local pharmacokinetic studies led us to design preclinical in vivo models resembling subtotal tumor resection surgery. Such surgical model is likely the most realistic approach to study the activity of local DDSs in pediatric oncology because in most patients, especially the ones with stage 4 neuroblastoma, tumors infiltrate or invade tissues surrounding vital vascular structures, a phenomenon that precludes complete tumor resection. Importantly, a recent study has reported on the activity of a doxorubicin-loaded local DDS to control neuroblastoma local recurrences after subtotal resection of orthotopic xenografts of neuroblastoma cell lines [46]. Because SN-38 is not in the standard of care regimen of the pediatric oncologic disease (as opposed to doxorubicin), our candidate DDS would provide an additional alternative in patients previously exposed to doxorubicin and showing resistance or tumor relapse. In addition, we performed all the in vivo activity studies in PDX models because they are likely more predictive of drug activity than cell lines and represent a first step towards a personalized therapy [47]. The Ewing sarcoma model HSJD-ES-001 is a subset of aggressive tumor with STAG2 and p53 mutation [48], whereas the neuroblastoma HSJD-NB-005 was derived from a stage 4 neuroblastoma tumor with amplification of MYCN and mutation of p53.

Our first set of in vivo studies in mice with bilateral tumors confirmed local activity of the DDS, because control tumors contralateral to the local DDS did not respond to local treatment upon subtotal bilateral resection (Figure 7). Such selective local activity is further supported by the finding of almost undetectable plasma SN-38 levels upon administration of the matrices, so that contralateral tumors are not exposed to SN-38 (Figures 4-6). The survival experiment on the neuroblastoma PDX model confirmed that the local DDS provided significant 
control of tumor recurrence, whilst equimolar injections of irinotecan (either local or systemic) did not perform better than the control treatment with blank matrices. Because a few tumors responded in the group receiving systemic irinotecan upon resection, we did not find a significant difference in survival between the group receiving SN-38 matrices and the one receiving systemic irinotecan. Nevertheless, systemic treatment immediately after resection is not a clinically acceptable practice because it interferes with the surgical recovery. In summary, we have addressed an unmet medical need in pediatric oncology by developing an advanced DDS that localizes the release of an anticancer drug with broad activity spectrum in pediatric cancers. We have also characterized local drug distribution, a procedure that we believe is critical to select the patients that would likely benefit of this novel therapeutic approach. Moreover, due to the potency of the model drug employed for the development, the system could be applicable to other oncologic diseases in which local control is crucial for the improvement of the therapeutic index. Ongoing work in the laboratory is focused on the activity of the new DDS on orthotopic pediatric tumor PDX models, and in the study of the systemic and local toxicology of the DDS upon administration next to vital organs like vessels, nerves and viscera.

\section{Acknowledgements}

AMC acknowledges funding from the AECC Scientific Foundation, MINECO (SAF2011-22660), Fundacion BBVA, European Union Seventh Framework Programme (FP7/2007-2013) under Marie Curie International Reintegration Grant (PIRG-08-GA-2010-276998) and ISCIII-FEDER (CP13/00189). AS thanks the Technion (grant \# 76535316). Work supported by the Xarxa de Bancs de 
Tumors de Catalunya (XBTC) sponsored by Pla Director d'Oncologia de Catalunya.

\section{References}

[1] Davidoff AM, Fernandez-Pineda I, Santana VM, Shochat SJ. The role of neoadjuvant chemotherapy in children with malignant solid tumors. Seminars in pediatric surgery. 2012;21:88-99.

[2] Kushner BH, Wolden S, LaQuaglia MP, Kramer K, Verbel D, Heller G, et al. Hyperfractionated low-dose radiotherapy for high-risk neuroblastoma after intensive chemotherapy and surgery. J Clin Oncol. 2001;19:2821-8.

[3] Rich BS, McEvoy MP, LaQuaglia MP, Wolden SL. Local control, survival, and operative morbidity and mortality after re-resection, and intraoperative radiation therapy for recurrent or persistent primary high-risk neuroblastoma. $\mathrm{J}$ Pediatr Surg. 2011;46:97-102.

[4] Rodriguez-Galindo C, Navid F, Liu T, Billups CA, Rao BN, Krasin MJ. Prognostic factors for local and distant control in Ewing sarcoma family of tumors. Ann Oncol. 2008;19:814-20.

[5] Mazzoleni S, Bisogno G, Garaventa A, Cecchetto G, Ferrari A, Sotti G, et al. Outcomes and prognostic factors after recurrence in children and adolescents with nonmetastatic rhabdomyosarcoma. Cancer. 2005;104:183-90.

[6] Rodriguez-Galindo C, Billups CA, Kun LE, Rao BN, Pratt CB, Merchant TE, et al. Survival after recurrence of Ewing Tumors. Cancer. 2002;94:561-9.

[7] Pui CH, Gajjar AJ, Kane JR, Qaddoumi IA, Pappo AS. Challenging issues in pediatric oncology. Nature reviews Clinical oncology. 2011;8:540-9.

[8] Kleinerman RA, Tucker MA, Abramson DH, Seddon JM, Tarone RE, Fraumeni JF, Jr. Risk of soft tissue sarcomas by individual subtype in survivors of hereditary retinoblastoma. J Natl Cancer Inst. 2007;99:24-31.

[9] Sosnik A, Carcaboso AM. Nanomedicines in the future of pediatric therapy. Adv Drug Deliv Rev. 2014;73C:140-61. 
[10] Wolinsky JB, Colson YL, Grinstaff MW. Local drug delivery strategies for cancer treatment: gels, nanoparticles, polymeric films, rods, and wafers. J Control Release. 2012;159:14-26.

[11] Moses MA, Brem H, Langer R. Advancing the field of drug delivery: taking aim at cancer. Cancer Cell. 2003;4:337-41.

[12] Thiagarajah JR, Kim JK, Magzoub M, Verkman AS. Slowed diffusion in tumors revealed by microfiberoptic epifluorescence photobleaching. Nature methods. 2006;3:275-80.

[13] Fung LK, Ewend MG, Sills A, Sipos EP, Thompson R, Watts M, et al. Pharmacokinetics of interstitial delivery of carmustine, 4hydroperoxycyclophosphamide, and paclitaxel from a biodegradable polymer implant in the monkey brain. Cancer Res. 1998;58:672-84.

[14] Arifin DY, Lee KY, Wang CH, Smith KA. Role of convective flow in carmustine delivery to a brain tumor. Pharm Res. 2009;26:2289-302.

[15] Monterrubio C, Paco S, Vila-Ubach M, Rodriguez E, Glisoni R, Lavarino C, et al. Combined Microdialysis-Tumor Homogenate Method for the Study of the Steady State Compartmental Distribution of a Hydrophobic Anticancer Drug in Patient-Derived Xenografts. Pharm Res. 2015;32:2889-900.

[16] Grohar PJ, Segars LE, Yeung C, Pommier Y, D'Incalci M, Mendoza A, et al. Dual targeting of EWS-FLI1 activity and the associated DNA damage response with trabectedin and SN38 synergistically inhibits Ewing sarcoma cell growth. Clin Cancer Res. 2014;20:1190-203.

[17] Thompson J, Zamboni WC, Cheshire PJ, Lutz L, Luo X, Li Y, et al. Efficacy of systemic administration of irinotecan against neuroblastoma xenografts. Clin Cancer Res. 1997;3:423-31.

[18] Stewart CF, Zamboni WC, Crom WR, Houghton PJ. Disposition of irinotecan and $\mathrm{SN}-38$ following oral and intravenous irinotecan dosing in mice. Cancer Chemother Pharmacol. 1997;40:259-65.

[19] Slatter JG, Schaaf LJ, Sams JP, Feenstra KL, Johnson MG, Bombardt PA, et al. Pharmacokinetics, metabolism, and excretion of irinotecan (CPT-11) 
following I.V. infusion of [(14)C]CPT-11 in cancer patients. Drug Metab Dispos. 2000;28:423-33.

[20] Koizumi F, Kitagawa M, Negishi T, Onda T, Matsumoto S, Hamaguchi T, et al. Novel SN-38-incorporating polymeric micelles, NK012, eradicate vascular endothelial growth factor-secreting bulky tumors. Cancer Res. 2006;66:1004856.

[21] Meinel AJ, Germershaus O, Luhmann T, Merkle HP, Meinel L. Electrospun matrices for localized drug delivery: current technologies and selected biomedical applications. European journal of pharmaceutics and biopharmaceutics : official journal of Arbeitsgemeinschaft fur Pharmazeutische Verfahrenstechnik eV. 2012;81:1-13.

[22] Ordonez JL, Amaral AT, Carcaboso AM, Herrero-Martin D, Del Carmen Garcia-Macias M, Sevillano V, et al. The PARP inhibitor olaparib enhances the sensitivity of ewing sarcoma to trabectedin. Oncotarget. 2015.

[23] Crompton BD, Stewart C, Taylor-Weiner A, Alexe G, Kurek KC, Calicchio $\mathrm{ML}$, et al. The genomic landscape of pediatric Ewing sarcoma. Cancer discovery. 2014;4:1326-41.

[24] Carcaboso AM, Elmeliegy MA, Shen J, Juel SJ, Zhang ZM, Calabrese C, et al. Tyrosine kinase inhibitor gefitinib enhances topotecan penetration of gliomas. Cancer Res. 2010;70:4499-508.

[25] Houghton PJ, Cheshire PJ, Hallman JC, Bissery MC, Mathieu-Boue A, Houghton JA. Therapeutic efficacy of the topoisomerase I inhibitor 7-ethyl-10(4-[1-piperidino]-1-piperidino)-carbonyloxy-camptothecin against human tumor xenografts: lack of cross-resistance in vivo in tumors with acquired resistance to the topoisomerase I inhibitor 9-dimethylaminomethyl-10-hydroxycamptothecin. Cancer Res. 1993;53:2823-9.

[26] Pappo AS, Lyden E, Breitfeld P, Donaldson SS, Wiener E, Parham D, et al. Two consecutive phase II window trials of irinotecan alone or in combination with vincristine for the treatment of metastatic rhabdomyosarcoma: the Children's Oncology Group. J Clin Oncol. 2007;25:362-9. 
[27] Morland B, Platt K, Whelan JS. A phase II window study of irinotecan (CPT11) in high risk Ewing sarcoma: a Euro-E.W.I.N.G. study. Pediatr Blood Cancer. 2014;61:442-5.

[28] Bagatell R, London WB, Wagner LM, Voss SD, Stewart CF, Maris JM, et al. Phase II Study of Irinotecan and Temozolomide in Children With Relapsed or Refractory Neuroblastoma: A Children's Oncology Group Study. J Clin Oncol. 2011;29:208-13.

[29] Xie M, Yang D, Wu M, Xue B, Yan B. Mouse liver and kidney carboxylesterase (M-LK) rapidly hydrolyzes antitumor prodrug irinotecan and the N-terminal three quarter sequence determines substrate selectivity. Drug Metab Dispos. 2003;31:21-7.

[30] Satoh T, Hosokawa M, Atsumi R, Suzuki W, Hakusui H, Nagai E. Metabolic activation of CPT-11, 7-ethyl-10-[4-(1-piperidino)-1piperidino]carbonyloxycamptothecin, a novel antitumor agent, by carboxylesterase. Biol Pharm Bull. 1994;17:662-4.

[31] Danks MK, Yoon KJ, Bush RA, Remack JS, Wierdl M, Tsurkan L, et al. Tumor-targeted enzyme/prodrug therapy mediates long-term disease-free survival of mice bearing disseminated neuroblastoma. Cancer Res. 2007;67:225.

[32] Bala V, Rao S, Boyd BJ, Prestidge CA. Prodrug and nanomedicine approaches for the delivery of the camptothecin analogue SN38. J Control Release. 2013;172:48-61.

[33] Falde EJ, Freedman JD, Herrera VL, Yohe ST, Colson YL, Grinstaff MW. Layered superhydrophobic meshes for controlled drug release. J Control Release. 2015;214:23-9.

[34] Xie C, Li X, Luo X, Yang Y, Cui W, Zou J, et al. Release modulation and cytotoxicity of hydroxycamptothecin-loaded electrospun fibers with 2hydroxypropyl-beta-cyclodextrin inoculations. Int J Pharm. 2010;391:55-64.

[35] Shenderova A, Burke TG, Schwendeman SP. The acidic microclimate in poly(lactide-co-glycolide) microspheres stabilizes camptothecins. Pharm Res. 1999;16:241-8. 
[36] Chang G, Ci T, Yu L, Ding J. Enhancement of the fraction of the active form of an antitumor drug topotecan via an injectable hydrogel. J Control Release. 2011;156:21-7.

[37] Wolinsky JB, Liu R, Walpole J, Chirieac LR, Colson YL, Grinstaff MW. Prevention of in vivo lung tumor growth by prolonged local delivery of hydroxycamptothecin using poly(ester-carbonate)-collagen composites. J Control Release. 2010;144:280-7.

[38] Ranganath SH, Fu Y, Arifin DY, Kee I, Zheng L, Lee HS, et al. The use of submicron/nanoscale PLGA implants to deliver paclitaxel with enhanced pharmacokinetics and therapeutic efficacy in intracranial glioblastoma in mice. Biomaterials. 2010;31:5199-207.

[39] Carcaboso AM, Bramuglia GF, Chantada GL, Fandino AC, Chiappetta DA, de Davila MT, et al. Topotecan vitreous levels after periocular or intravenous delivery in rabbits: an alternative for retinoblastoma chemotherapy. Invest Ophthalmol Vis Sci. 2007;48:3761-7.

[40] Carcaboso AM, Chiappetta DA, Opezzo JA, Hocht C, Fandino AC, Croxatto $\mathrm{JO}$, et al. Episcleral implants for topotecan delivery to the posterior segment of the eye. Invest Ophthalmol Vis Sci. 2010;51:2126-34.

[41] Ranganath $\mathrm{SH}$, Wang $\mathrm{CH}$. Biodegradable microfiber implants delivering paclitaxel for post-surgical chemotherapy against malignant glioma. Biomaterials. 2008;29:2996-3003.

[42] Zong S, Wang X, Yang Y, Wu W, Li H, Ma Y, et al. The use of cisplatinloaded mucoadhesive nanofibers for local chemotherapy of cervical cancers in mice. European journal of pharmaceutics and biopharmaceutics : official journal of Arbeitsgemeinschaft fur Pharmazeutische Verfahrenstechnik eV. 2015;93:127-35.

[43] Liu R, Wolinsky JB, Catalano PJ, Chirieac LR, Wagner AJ, Grinstaff MW, et al. Paclitaxel-eluting polymer film reduces locoregional recurrence and improves survival in a recurrent sarcoma model: a novel investigational therapy. Ann Surg Oncol. 2012;19:199-206. 
[44] Liu R, Wolinsky JB, Walpole J, Southard E, Chirieac LR, Grinstaff MW, et al. Prevention of local tumor recurrence following surgery using low-dose chemotherapeutic polymer films. Ann Surg Oncol. 2010;17:1203-13.

[45] Liu S, Wang X, Zhang Z, Zhang Y, Zhou G, Huang Y, et al. Use of asymmetric multilayer polylactide nanofiber mats in controlled release of drugs and prevention of liver cancer recurrence after surgery in mice. Nanomedicine. 2015;11:1047-56.

[46] Chiu B, Coburn J, Pilichowska M, Holcroft C, Seib FP, Charest A, et al. Surgery combined with controlled-release doxorubicin silk films as a treatment strategy in an orthotopic neuroblastoma mouse model. $\mathrm{Br} \mathrm{J}$ Cancer. 2014;111:708-15.

[47] Hidalgo M, Amant F, Biankin AV, Budinska E, Byrne AT, Caldas C, et al. Patient-derived xenograft models: an emerging platform for translational cancer research. Cancer discovery. 2014;4:998-1013.

[48] Tirode F, Surdez D, Ma X, Parker M, Le Deley MC, Bahrami A, et al. Genomic landscape of Ewing sarcoma defines an aggressive subtype with coassociation of STAG2 and TP53 mutations. Cancer discovery. 2014;4:1342-53. 
Table 1. Clinical details of the PDX models.

\begin{tabular}{|c|c|c|c|c|c|c|c|c|}
\hline $\begin{array}{l}\text { Model } \\
\text { code }\end{array}$ & $\begin{array}{l}\text { Source of } \\
\text { biopsy }^{1}\end{array}$ & $\begin{array}{l}\text { Age at } \\
\text { biopsy }\end{array}$ & $\begin{array}{l}\text { Primary } \\
\text { tumor }\end{array}$ & $\begin{array}{c}\text { Age at } \\
\text { diagnosis }\end{array}$ & $\begin{array}{c}\text { Metastasis at } \\
\text { diagnosis }\end{array}$ & Demography & Tumor properties & $\begin{array}{l}\text { Patient } \\
\text { status }\end{array}$ \\
\hline $\begin{array}{l}\text { HSJD- } \\
\text { NB-005 }\end{array}$ & $\begin{array}{l}\text { Tumor refractory } \\
\text { to treatment } \\
\text { (metastasis in } \\
\text { bone marrow) }\end{array}$ & $2.5 y$ & $\begin{array}{l}\text { Mass } \\
\text { arising from } \\
\text { kidney }\end{array}$ & $2.0 \mathrm{y}$ & $\begin{array}{l}\text { Yes (ganglia, } \\
\text { bone marrow, } \\
\text { lungs) }\end{array}$ & Female, white & $\begin{array}{c}\text { Stage } 4 \text { neuroblastoma, } \\
\text { MYCN amplified, P53 } \\
\text { mutation }\end{array}$ & $\begin{array}{l}\text { Died of } \\
\text { disease }\end{array}$ \\
\hline $\begin{array}{l}\text { HSJD- } \\
\text { ES-001 }\end{array}$ & $\begin{array}{l}\text { Local relapse in } \\
\text { scapula }^{2}\end{array}$ & $21.7 y$ & Scapula & $17 y$ & $\begin{array}{l}\text { Yes (lungs, } \\
\text { bone, bone } \\
\text { marrow) }\end{array}$ & Male, white & $\begin{array}{l}\text { EWS-FLI1 fusion gene, } \\
\text { STAG2 mutation, P53 } \\
\text { mutation }\end{array}$ & $\begin{array}{l}\text { Died of } \\
\text { disease }\end{array}$ \\
\hline
\end{tabular}

${ }^{1}$ Tumor tissue was collected with informed consent under an Institutional Review Board-approved protocol.

${ }^{2}$ Further details on this tumor are published in [23], named after the code SJDES023. 
Table 2. Antiproliferative activity of SN-38 (stock solution in DMSO) against pediatric solid tumor models.

\begin{tabular}{|l|l|l|l|}
\hline Model & Properties & IC50 (nM) & $\mathbf{9 5 \%}$ confidence interval (nM) \\
\hline LAN-1 & NB cell line & 60.8 & $33.1-111$ \\
\hline SK-N-AS & NB cell line & 215 & $162-284$ \\
\hline HSJD-NB-005 & NB PDX primary culture & 25.2 & $17.1-37.1$ \\
\hline SK-ES-1 & ES cell line & 0.927 & $0.747-1.15$ \\
\hline HSJD-ES-001 & ES PDX primary culture & 1.48 & $1.06-2.06$ \\
\hline Rh30 & aRMS cell line & 7.57 & $4.62-12.4$ \\
\hline
\end{tabular}

NB: neuroblastoma; ES: Ewing sarcoma; aRMS: alveolar rhabdomyosarcoma 